Research Article

\title{
Neural Network Segmentation Algorithm-Based Magnetic Resonance Imaging to Explore the Relationship between Cerebrospinal Fluid Flow with Communicating Hydrocephalus after Decompressive Craniectomy for Craniocerebral Injury
}

\author{
Yan Wang $\mathbb{D},{ }^{1}$ Yanmin Zhang $\mathbb{D},{ }^{1}$ and Chunyue Zhang $\mathbb{D}^{2}$ \\ ${ }^{1}$ Department of Neurosurgery, Hengshui People's Hospital, Hengshui,053000, Hebei, China \\ ${ }^{2}$ Department of Oncology, Hengshui People's Hospital, Hengshui,053000, Hebei, China \\ Correspondence should be addressed to Yan Wang; 1420040140@st.usst.edu.cn
}

Received 7 September 2021; Revised 31 October 2021; Accepted 5 November 2021; Published 24 November 2021

Academic Editor: M Pallikonda Rajasekaran

Copyright $\odot 2021$ Yan Wang et al. This is an open access article distributed under the Creative Commons Attribution License, which permits unrestricted use, distribution, and reproduction in any medium, provided the original work is properly cited.

\begin{abstract}
This study aimed to explore the application value of magnetic resonance imaging optimized by neural network segmentation algorithm in analyzing the relationship between cerebrospinal fluid changes after decompressive craniectomy and the occurrence of communicating hydrocephalus. 100 patients with craniocerebral injury undergoing decompressive craniectomy in hospital were selected as research subjects. The collected MRI images were processed using the OTSU algorithm, the cerebrospinal fluid flow rate was calculated based on the observation results, and the MRI based on the neural network segmentation algorithm was used to analyze the relationship between the occurrence of communicating hydrocephalus with the cerebrospinal fluid flow after decompressive craniectomy for craniocerebral injury. Additionally, the dynamics of the flow of cerebrospinal fluid in the midbrain aqueduct was analyzed. After decompressive craniectomy for craniocerebral injury, of the 24 cases of cerebrospinal fluid accumulation, 23 cases had hydrocephalus; of the 55 cases of cerebrospinal fluid flow disorder, hydrocephalus occurred in 47 cases; and of the 21 cases of normal cerebrospinal fluid, no patients had hydrocephalus. For patients with communicating hydrocephalus, the cerebrospinal fluid flow at the aqueduct was obviously accelerated and the flow was increased. From this, the differential diagnosis of cerebrospinal fluid and communicating hydrocephalus can be further confirmed. The results showed that the third ventricle of the study group was significantly reduced, and the flow of cerebrospinal fluid was similar to that of normal people. It suggested that decompressive craniectomy can relieve communicating hydrocephalus. In patients with communicating hydrocephalus, the cerebrospinal fluid flow at the aqueduct was significantly accelerated, the flow amount was increased, and the blocked flow of cerebrospinal fluid can also lead to hydrocephalus, which further clarified the relationship between the occurrence of communicating hydrocephalus with the flow of cerebrospinal fluid. In short, the neural network segmentation algorithm-based magnetic resonance imaging demonstrated a good value in the analysis of craniocerebral injury, from which the doctor observed that the cerebrospinal fluid flow at the aqueduct was significantly accelerated. Its detection of brain complications after decompressive craniectomy was also effective.
\end{abstract}

\section{Introduction}

Craniocerebral injury not only causes brain damage but also affects the whole body. Its mortality and disability rates are much higher than other diseases. Investigations have shown that the annual incidence of traumatic head and cervical spine injuries reaches $0.001 \%$ [1]. Since the last century, the mortality of patients with craniocerebral injury has been significantly reduced with the continuous improvement of medical technology, but the mortality rate is still high [2]. At present, surgical intervention is the main treatment for patients with craniocerebral injury [3]. For severely ill 
patients with acute intracranial hypertension, decompressive craniectomy can quickly relieve malignant intracerebral hypertension, reduce the incidence of brain pressure-induced, and improve the state of high cerebrospinal fluid flow [4]. However, surgical intervention can cause changes in intracranial blood, cerebral fluid circulation, and microvascular structure, resulting in complicated postoperative complications. There have been studies evaluating the effect of decompression craniectomy. The main content is to explore the relationship between the treatment effect and surgical complications [5].

Hydrocephalus is a common complication of craniocerebral injury. Additionally, brain infections and brain tumors can also cause hydrocephalus in fetuses, and it mostly manifests as malabsorption of cerebrospinal fluid. Clinically, hydrocephalus can be divided into two types: communicating hydrocephalus and obstructive hydrocephalus [6]. The communicating hydrocephalus arises from malabsorption of cerebrospinal fluid and communicating block [7]. The occlusive hydrocephalus is identified based on the location inside and outside the ventricle. During the formation of hydrocephalus, ventricular dilation blocks the flow of cerebrospinal fluid, resulting in irreversible hydrocephalus. As the disease progresses, intracranial pressure rises, blood flow is blocked, and the brain shrinks. It is very meaningful to evaluate the postoperative effect of hydrocephalus. Hydrocephalus is caused by abnormal accumulation of cerebrospinal fluid, which increases intracranial pressure. When hydrocephalus occurs, the patient's systemic function is impaired, and the patient is unable to live and communicate normally. It will also cause permanent damage to the patient's physical and mental health [8].

Hydrocephalus under normal circumstances disrupts the balance of cerebrospinal fluid, eventually leading to accumulation of cerebrospinal fluid and swelling of the ventricles. For congenital hydrocephalus, the patient's head is significantly enlarged, and it can be identified by observing this external feature [9]. When hydrocephalus occurs in adults, the cranial volume does not change significantly, so doctors need to analyze medical images to diagnose hydrocephalus $[10,11]$. During the process, the doctor needs to observe the structure of each layer of MRI images. With the advent of computer-aided diagnosis, it has assisted physicians in diagnosing many brain diseases, such as brain tumors and cerebral hemorrhage, stroke, and cerebral infarction [12]. The segmentation of contralateral ventricle is usually based on regional segmentation due to the obvious regional distribution of brain medical images. Affected by noise, the MR image shows that the ventricle appears to be connected to the cerebrospinal fluid. Nowadays, magnetic resonance imaging (MRI) analysis based on the neural network segmentation algorithm is applied to the diagnosis of hydrocephalus. Hydrocephalus can cause the patient's ventricles to expand, but the volume of the cranial cavity does not change significantly, and thus the volume ratio of the ventricle to the cranial cavity may exceed the normal range in hydrocephalus patients [13]. Generally speaking, the diagnosis of hydrocephalus requires volume information of the ventricles and cranial cavity. The structure of the cranial cavity is relatively simple, and it is easy to disassemble, while the structure of the lateral ventricle is complicated. Therefore, the main task is to segment the lateral ventricle to diagnose hydrocephalus [14-17].

In the past few years, the MRI has been developed to study the physiology of cerebrospinal fluid circulation and diagnose the hydrocephalus. In this study, neural network segmentation algorithm-based MRI was used to analyze the relationship between changes in cerebrospinal fluid and communicating hydrocephalus after decompressive craniectomy for craniocerebral injury.

\section{Materials and Methods}

2.1. Research Subjects. In the study, 100 patients with craniocerebral injury admitted to hospital from May 2019 to May 2021 were selected as research subjects. This study has been approved by the Medical Ethics Committee of Hospital, and the family members of the patients included in the study had signed an informed consent form.

Inclusion criteria are as follows: (I) hydrocephalus patients caused by craniocerebral injury, and no clear cerebrospinal fluid flow could be observed in the midbrain aqueduct area; (II) cerebrospinal fluid area could be observed by MRI brain imaging; (III) patients not less than 25 years old; and (IV) patients not accompanied by other brain diseases.

Exclusion criteria are as follows: (1) previous history of decompressive craniectomy, bilateral decompressive craniectomy, and posterior fossa decompression; (2) previous history of other neurological diseases, such as brain tumor, meningitis, and stroke; (3) patients with poor compliance; (4) patients suffering from other brain diseases or hydrocephalus removal complications (such as cerebral hemorrhage and encephalitis); (5) the first CT examination indicated previous hydrocephalus or cerebral hydrocephalus circulation obstruction; and (6) with open craniocerebral injury.

2.2. MRI Examination for Brain. MRI, CT, and ultrasound are widely used in clinical diagnosis of diseases. MRI, as one of the medical imaging technologies, uses magnetic fields, electric waves, and gradients to generate medical images of internal tissue in the body. The hydrogen nuclei in various tissue of the human body can be excited by atoms, electromagnetic waves, and so on, and the energy attenuation can be converted to computer signals to generate MRI images [18]. Figure 1 shows the magnetic resonance imaging system.

MR images can be divided into different types based on relaxation times. T1- and T2- weighted sequence images are commonly used. As shown in Figure 2, Figure 2(a) is the T1weighted sequence image of the brain and Figure 2(b) is the T2-weighted sequence image of the brain.

T1 emphasizes the difference in the longitudinal relaxation of the tissue in the image. It is suitable for observing anatomical structures. The intracranial tissue in MRI images mainly includes cerebrospinal fluid, white matter, and gray 


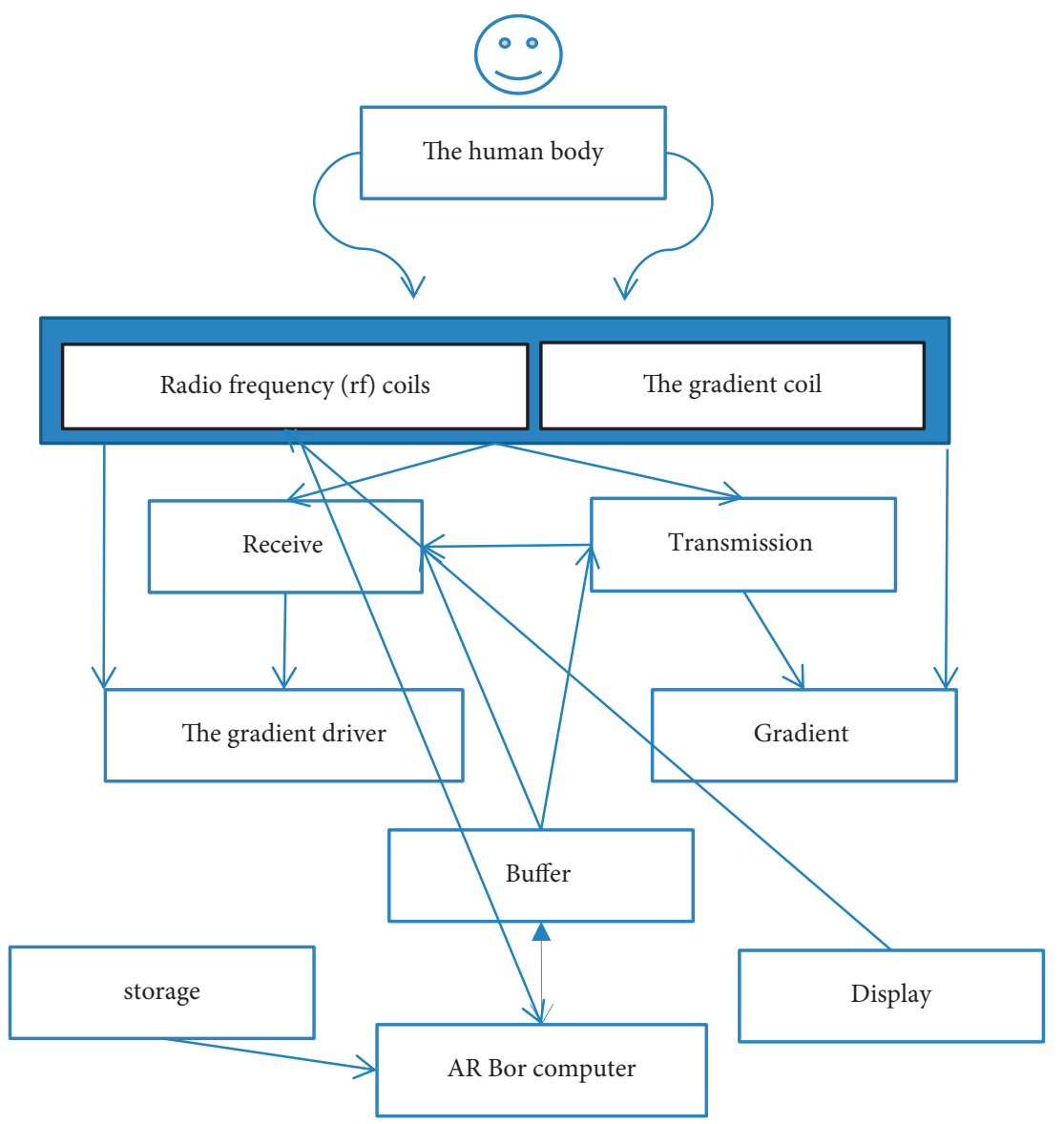

Figure 1: The MRI system.

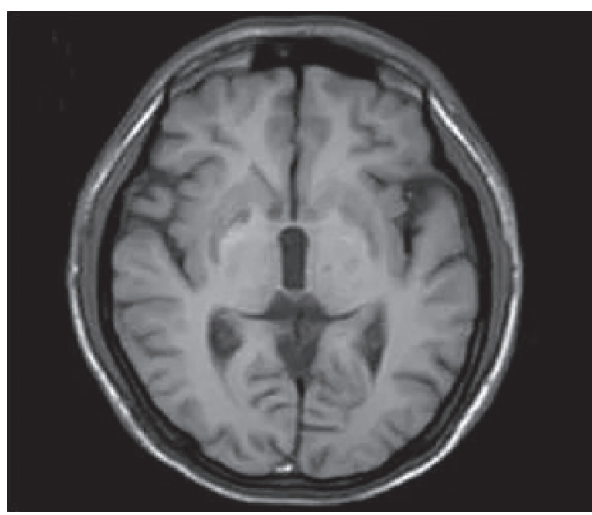

(a)

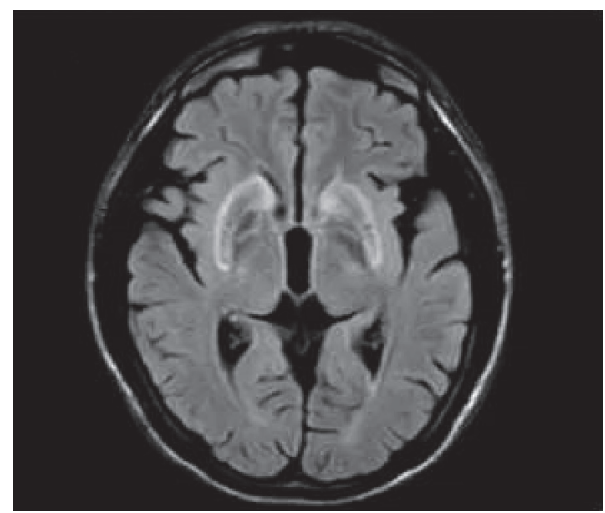

(b)

FIGURE 2: MRI images: (a) the T1-weighted sequence image of the brain and (b) the T2-weighted sequence image of the brain.

matter. In the same tissue, the gray values of corresponding pixels on the image are not necessarily in the same range. There may exist artifacts, and the edges of the image are not clear, and the lesions are not obvious.

2.3. The Auxiliary Diagnosis of Hydrocephalus Based on Ventricle Segmentation. As shown in Figure 3, the auxiliary diagnosis process of hydrocephalus can be divided into three steps, namely, preprocessing, segmentation, and result generation. The existence of noise increases the difficulty of image segmentation and image analysis. Therefore, it is necessary to process the image first to improve the accuracy and precision. The auxiliary diagnosis of hydrocephalus needs to take the volume ratio of the ventricle and the cranial cavity as the reference value. Therefore, it is necessary to segment the ventricle and the cranial cavity. 


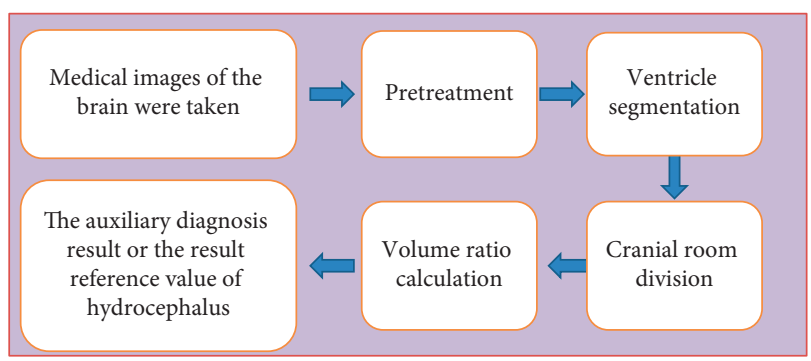

FIGURE 3: Flowchart of auxiliary diagnosis of hydrocephalus by segmenting ventricles.

2.4. Preprocessing. The quality of medical images is affected by many factors, such as the image capture device, the environment, and the subtle movements of the patient. Usually, most of the preprocessing includes noise removal, uneven field correction, registration, and skull anatomy. The main purpose of preprocessing is to reduce the influence of image noise. There are three main calculation methods using artificial intelligence algorithms: mean filtering, median filtering, and adaptive filtering. The study focuses on the mean filtering. The mean filtering is a single linear filter, and the average value is used to represent the surrounding pixels, as shown in the following:

$$
g(x, y)=\frac{1}{M} \sum_{f \in s} f(x, y) .
$$

Above, the average value of the mean filter can quickly get the result and remove the noise, but when it encounters the pixels with large differences, the details cannot be well preserved. However, it is efficient and fast and widely used. Figure 4 shows image processing results by the 3 methods.

2.5. Segmentation of MRI Images of Brain. Medical image segmentation is to segment the image into different areas according to the gray value, gray gradient, texture, and other characteristics of the image and extract the lesion area from the image. The segmentation of the ventricle and the cranial cavity is based on the gray contrast between the tissue. As shown in Figure 5, the lateral ventricle has a three-dimensional structure. In the figure, arrow 2 refers to the lower part of the lateral ventricle. The segmentation of lateral ventricle is prone to errors, and part of the lateral ventricular structure penetrates. This has brought about great difficulties recognizing images using naked eyes, but artificial intelligence is sensitive to subtle grayscale differences and can accurately recognize [19].

2.6. Auxiliary Diagnosis Results. After the brain image is segmented, the data structure can be read using software. Then, the cranial cavity volume can be calculated, and then the ratio of the ventricle to the cranial cavity volume can be calculated. For adults, cerebrospinal fluid accumulates, causing the ventricle to expand. Therefore, the volume ratio of ventricle to cranial cavity of hydrocephalus patients is relatively large, and the patient can be diagnosed based on the index.
2.7. OTSU Algorithm. The OTSU algorithm can obtain the threshold value through iterative calculation, and the binarization processing of the grayscale image is performed based on the threshold value [20]. The algorithm is to find the best threshold in the binarized image and obtain the smallest intraclass variance. The exhaustive search method based on the Otsu algorithm can obtain the minimum intraclass variance threshold, which is defined as the weighted sum of the variances of the two classes.

$$
\sigma_{\omega}^{2}(t)=\omega_{0}(t) \sigma_{0}^{2}(t)+\omega_{1}(t) \sigma_{1}^{2}(t) .
$$

The weight $\omega_{1}$ is the probability of the two classes separated by the threshold $t$, but $\sigma_{\omega}^{2}$ is the variance of the two classes, expressed as follows:

$$
\begin{aligned}
& \omega_{0}(t)=\sum_{i=0}^{t-1} p(i), \\
& \omega_{1}(t)=\sum_{i=l}^{L-1} p(i) .
\end{aligned}
$$

OTSU proves that minimizing the intraclass variance is equivalent to maximizing the between-class variance. The class probability $\omega_{1}$ and class mean $\mu_{1}$ represent the variance between the two classes, expressed as follows:

$$
\begin{aligned}
\sigma_{b}^{2}(t) & =\omega_{0}\left(\mu_{0}-\mu_{r}\right)^{2}+\omega_{1}\left(\mu_{1}-\mu_{r}\right)^{2} \\
& =\omega_{0}(t) \omega_{1}(t)\left[\mu_{0}(t)-\mu_{1}(t)\right]^{2} .
\end{aligned}
$$

The mean value is calculated as the following equations:

$$
\begin{aligned}
\mu_{0}(t) & =\sum_{i=0}^{i-1} i \frac{p(i)}{\omega_{0}}, \\
\mu_{1}(t) & =\sum_{i=l}^{L-1} i \frac{p(i)}{\omega_{1}} \\
\mu_{T} & =\sum_{i=0}^{L-1} i p(i) .
\end{aligned}
$$

The relationship between the probability of the class and the variance of the class is shown in the following equation:

$$
\begin{gathered}
\omega_{o} \mu_{o}+\omega_{1} \mu_{1}=\mu_{T}, \\
\omega_{o}+\omega_{1}=1 .
\end{gathered}
$$

2.8. Imaging Examination. The MRI routine scanning: head transverse axis and coronal SETIWI, TR is set to 360-450 $\mathrm{ms}$, TE is $12 \mathrm{~ms}$, FOV is $240-280 \mathrm{~mm}$, and matrix is $256 \times 217$. Head transverse axis TSE T2W1: TR is $4000 \mathrm{~ms}$, TE is 100 $\mathrm{ms}$, FOV is $250 \mathrm{~mm}$, and matrix is $256 \times 240$. The layer thickness of all the above sequences is $6 \mathrm{~mm}$, and the layerto-layer spacing is $0.6 \mathrm{~mm}$. The high-resolution sagittal SE T1W1: TR is $500 \mathrm{~ms}$, TE is $12 \mathrm{~ms}$, FOV is $180 \mathrm{rnm}$, the layer thickness of each sequence is $3 \mathrm{~mm}$, the layer-to-layer spacing is $0.3 \mathrm{~mm}$, and the matrix is $512 \times 2560$. All subjects 


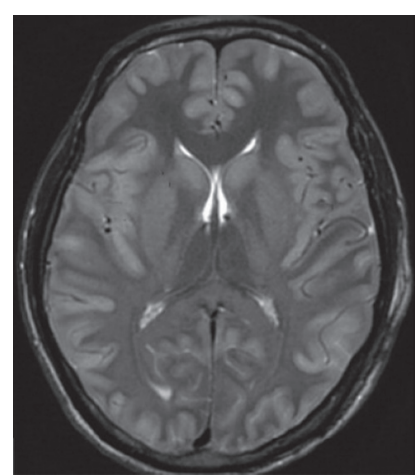

(a)

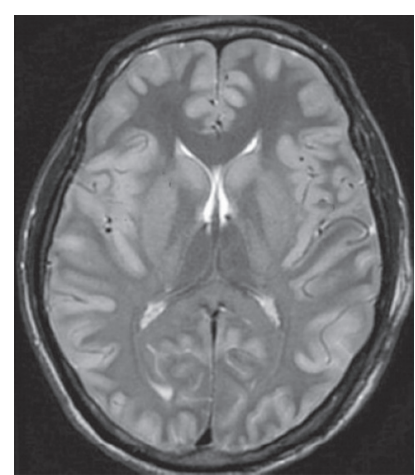

(b)

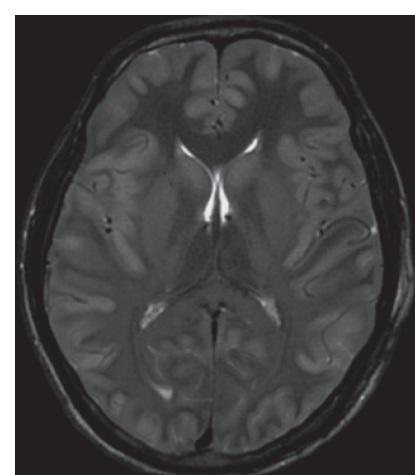

(c)

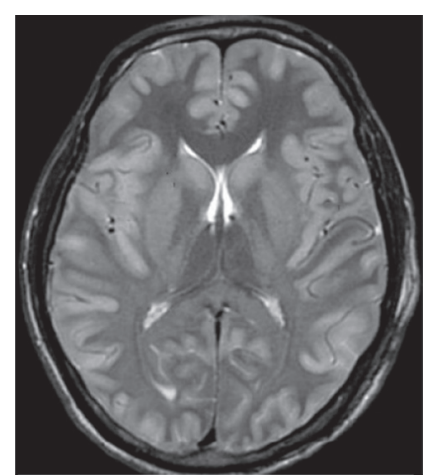

(d)

FIGURE 4: Image processing results of mean filter, median filter, and adaptive filter: (a) original image; (b) mean filter processed image; (c) median filter processed image; (d) adaptive filter processed image.

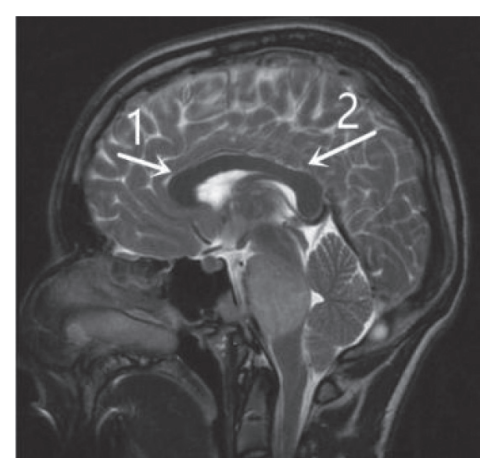

FIGURE 5: The upper and lower parts of the lateral ventricle.

had the phase-contrast MRI. First, the midsagittal film is collected. TR is $32.7 \mathrm{~ms}$, TE is $10.0 \mathrm{~ms}$, flip angle is $10^{\circ}$, FOV is $135 \mathrm{~mm} \times 135 \mathrm{~mm}$, the layer thickness of each sequence is $5 \mathrm{~mm}$, the flow velocity is $12 \mathrm{~cm} / \mathrm{s}$, and the number of image phases collected in each cardiac cycle is 9 frames.

2.9. Data Analysis. The flow fluid analysis software was used to measure and analyze the CSF flow in the corresponding areas in the study group and the control group. First, the collected images are enlarged to 4 images per screen, and then the elliptical region of interest (ROI) was used to delineate the outline of corresponding areas in the study group and the control group. To avoid subjective differences, two doctors (different age and seniority) described the ROI independently. The maximum systolic velocity (MSV) and the maximum diastolic velocity (MDV) were recorded, and then the average flow velocity was calculated.

2.10. Statistical Methods. SPSS20.0 was used for analysis and statistics. Normally distributed measurement data were expressed as mean \pm standard deviation. One-way analysis of variance was used for comparison between groups; independent $t$-test was used for general data, and paired sample $t$-test was used to compare LUS scores of the lungs. $P<0.05$ was the threshold for significance.

\section{Results}

3.1. The Accuracy of Segmentation. The segmentation results of the neural network segmentation algorithm were compared with the doctor's manual segmentation results, as shown in Figure 6. First, in order to show the accuracy of different segmentation methods, representative images of the upper, middle, and lower parts of the lateral ventricle were selected for comparison. The upper part is relatively simple, including two parts of the lateral ventricle, the middle part includes the third ventricle, and the lower part includes a large amount of nonlateral ventricle cerebrospinal fluid. The images from left to right were the original image, the image segmented by the physician, and the image segmented by the neural network. It was noted that for the upper, middle, and lower parts, the neural network segmentation results were close to the manual segmentation results. The image segmented by the neural network was clear, and the saturation was high.

3.2. Comparison of Image Gray Values. Three algorithms were used to denoise the brain image. The results are shown in Figure 7. Compared with the original image, the denoising image by median filtering was fuzzy, affecting the segmentation accuracy of the lateral ventricle. The denoising images by mean filtering and adaptive filtering were clear and close to the original grayscale image. The smooth mean filter curve indicated that the image quality was uniform.

3.3. Brain MRI Images and Cerebrospinal Fluid Flow. As shown in Figure 8, the preoperative MRI image of the study group showed that the lateral ventricle and the third ventricle were significantly enlarged, the midbrain aqueduct was narrowed or completely obstructed, the cerebrospinal fluid flow waveform was disordered (sometimes linear), and there was no obvious indication of partial or complete obstruction. The postoperative MRI image showed that the third ventricle was significantly reduced, and the flow of cerebrospinal fluid showed a bidirectional flow similar to that of normal people (Figure 9 and10). 

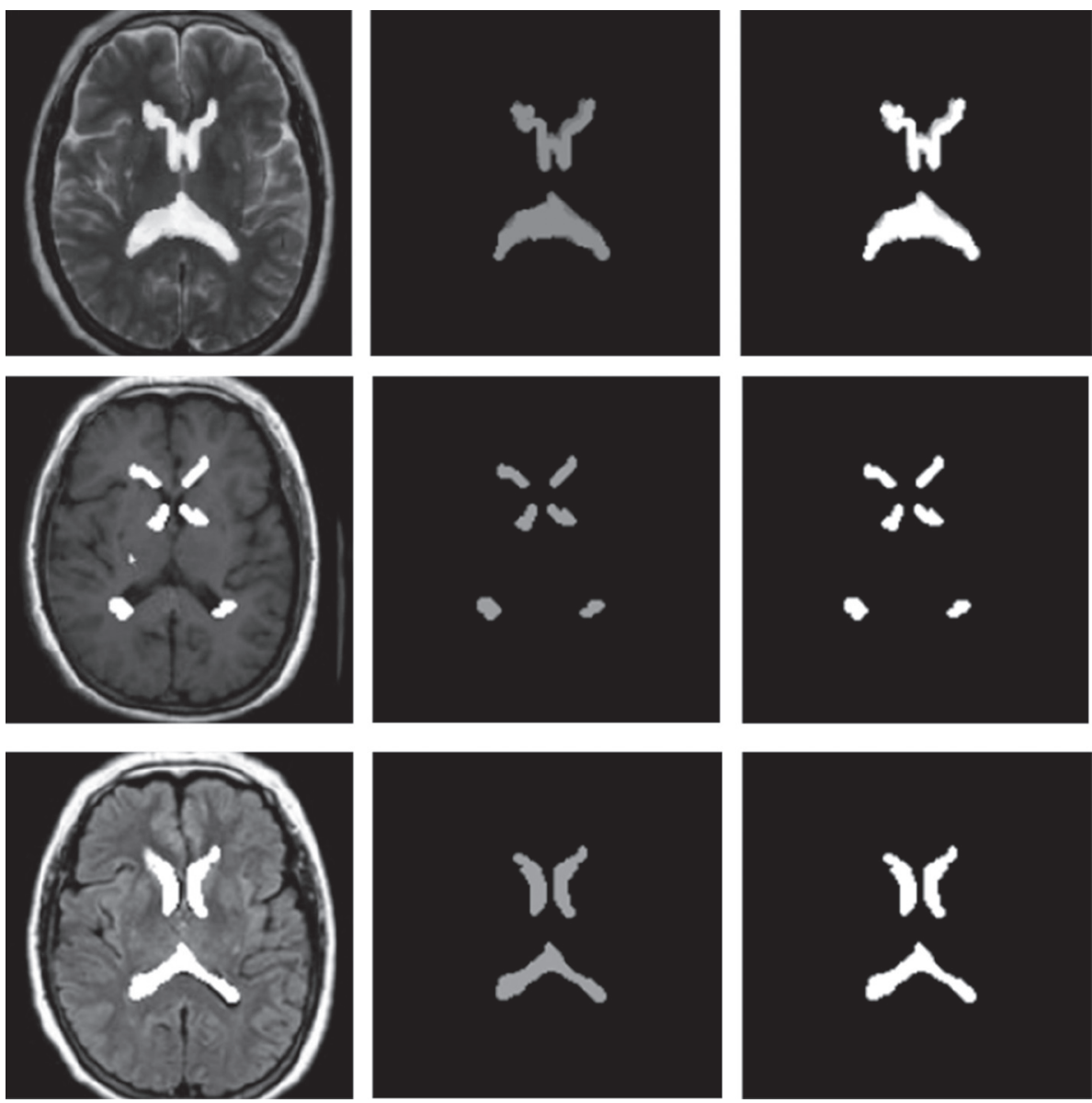

Figure 6: Comparison of segmentation results by the neural network and by physicians. (the segmentation results of the upper, middle, and lower parts of the lateral ventricle).

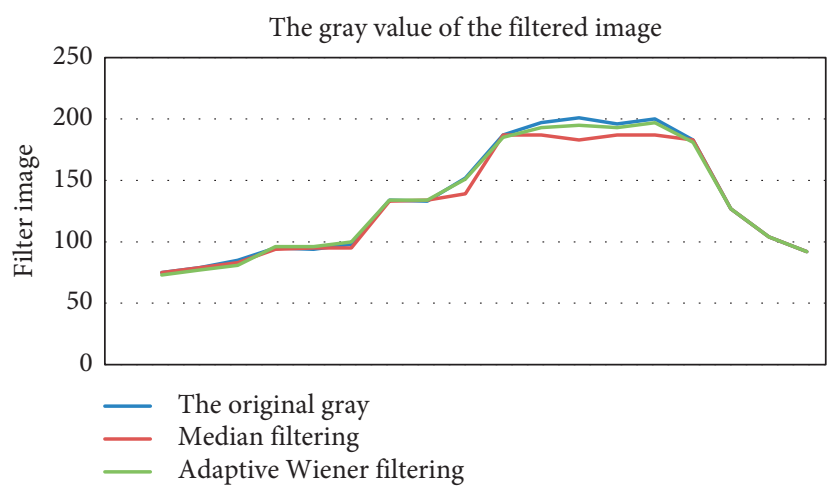

Figure 7: The gray value of the filtered image.

3.4. The Relationship between Cerebrospinal Fluid Flow and Hydrocephalus. As shown in Figure 11, of the 24 cases of cerebrospinal fluid accumulation, 23 cases had hydrocephalus; of the 55 cases of cerebrospinal fluid flow disorder, hydrocephalus occurred in 47 cases; and of the 21 cases of normal cerebrospinal fluid, no patients had hydrocephalus $(P<0.05)$. The flow of cerebrospinal fluid at the aqueduct was significantly accelerated, and the flow amount was increased in patients with communicating hydrocephalus. It clarified the relationship between cerebrospinal fluid and communicating hydrocephalus.

\section{Discussion}

Hydrocephalus commonly arises from meningitis, brain trauma, subarachnoid hemorrhage, and intracranial lesions after surgery. It can also be caused by abnormal secretion of the choroid plexus and narrow or occlusion of the 


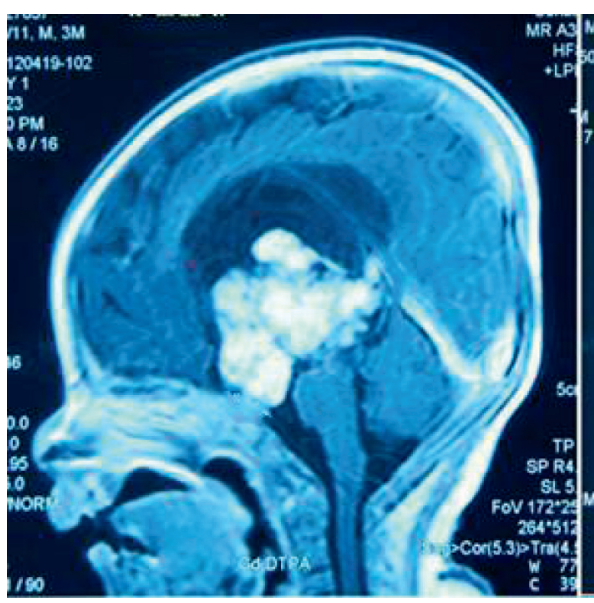

(a)

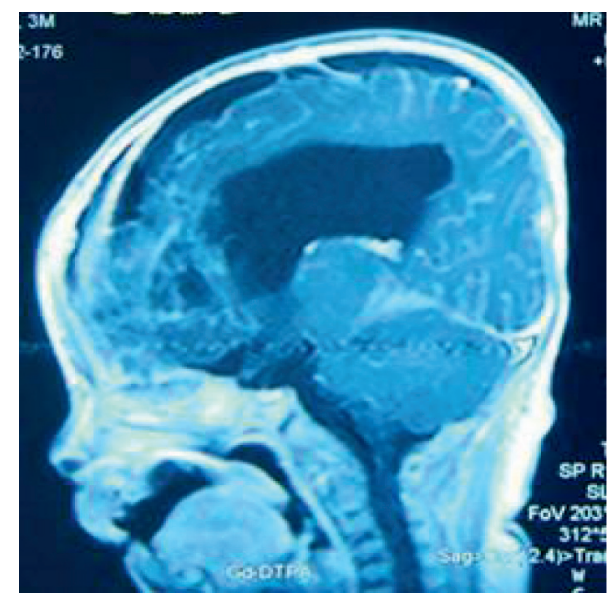

(b)

FIgURE 8: MRI images of the brain (a) before surgery and (b) after surgery.

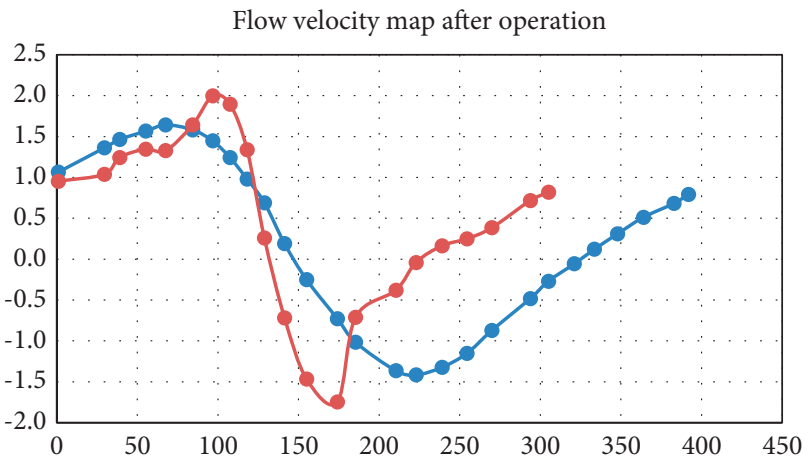

FIGURE 9: Flow velocity wave of cerebrospinal fluid before surgery.

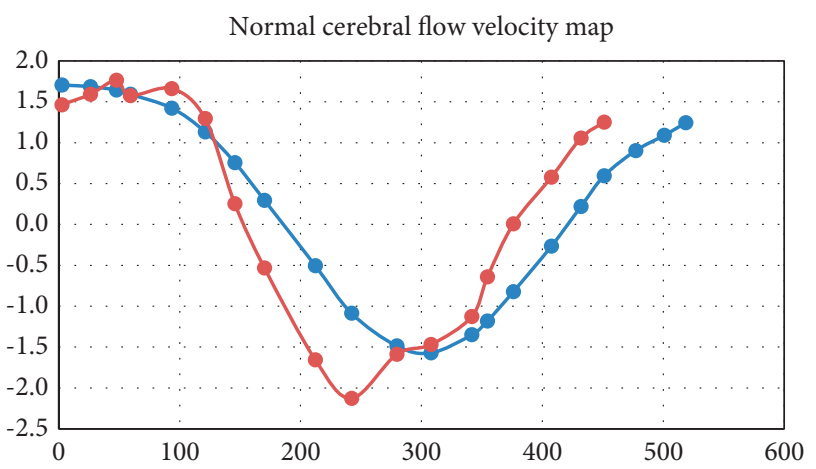

FIGURE 10: Flow velocity wave of cerebrospinal fluid after surgery.

intracranial venous cavity. Clinically, it is divided into two types of communicative and obstructive hydrocephalus. The former is due to the cerebrospinal fluid disorder or the absorption of arachnoid particles in the cerebrospinal fluid, but there is no obstacle between the cerebrovascular and the subarachnoid space, and it remains unobstructed. The latter is due to occlusion (stenosis or occlusion) of part of the cerebrospinal fluid circulation, which is often accompanied by ventricular dilatation, in which it is difficult to diagnose [21]. Due to the small diameter of the brain, it is difficult to

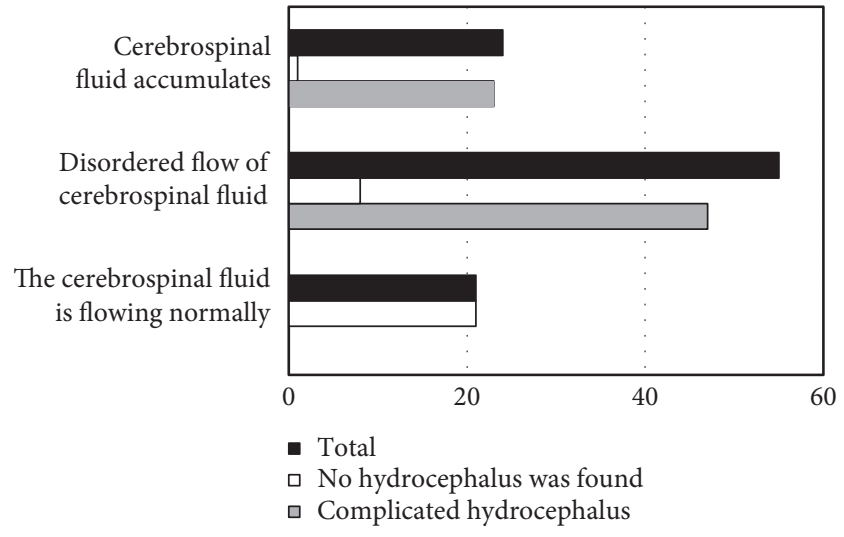

FIgURE 11: The relationship between the flow of cerebrospinal fluid and the occurrence of hydrocephalus.

determine whether the water channel is narrow or not using general imaging images such as static CT or MRI [22]. However, MRI is highly sensitive to fluid flow and can dynamically show even slight flow in the cerebrospinal fluid circulation path. Therefore, MRI has a strong advantage in identifying whether the cerebrospinal fluid circulation path is blocked or the degree of failure. Occlusive hydrocephalus includes partially occlusive hydrocephalus and completely occlusive hydrocephalus. In patients with partially occlusive hydrocephalus, according to the phase image, the cerebrospinal fluid shows a high signal in the systolic phase and a low signal in the dilated phase. In patients with completely occlusive hydrocephalus, the flow rate and flow amount of the cerebrospinal fluid are reduced. At this time, MRI is not sensitive to tissue and fluids, and water pipes and peripheral brain tissue cannot be distinguished by phase images, and thus the flow of brain fluid cannot be observed [23]. In patients with communicating hydrocephalus, the phase image of midbrain shows that the flow rate is 10 times that of normal people. Therefore, MRI image is a good way to distinguish communicating hydrocephalus from noncommunicating hydrocephalus. Godenschweger et al. (2016) 
studied the dynamics of cerebrospinal fluid circulation using MRI technology [24]. They observed the changes of cerebrospinal fluid signal intensity in different cardiac cycles, but they did not further study the pathological physiology of cerebrospinal fluid circulation dynamics. After decompressive craniectomy for craniocerebral injury, of the 24 cases of cerebrospinal fluid accumulation, 23 cases had hydrocephalus; of the 55 cases of cerebrospinal fluid flow disorder, hydrocephalus occurred in 47 cases; and of the 21 cases of normal cerebrospinal fluid, no patients had hydrocephalus. For patients with communicating hydrocephalus, the cerebrospinal fluid flow at the aqueduct was obviously accelerated and the flow was increased. From this, the differential diagnosis of cerebrospinal fluid and communicating hydrocephalus can be further confirmed. Postoperative cerebrospinal fluid flow wave reached the peak at 100 , and the lowest at about 220. After the surgery, MRI examination of cerebrospinal fluid was performed to explore the dynamics of cerebrospinal fluid flow in the midbrain aqueduct. The results showed that the third ventricle was significantly reduced, and the cerebrospinal fluid flow showed the normal bidirectional flow.

In the study, the characteristics of brain MRI images were analyzed first. Next, the basic steps of the auxiliary diagnosis of hydrocephalus were expounded. Then, the flow waveform of the cerebrospinal fluid in the hydrocephalus patients was studied, and the MRI image of the hydrocephalus was analyzed to provide help for the patient's future treatment. Disadvantages of this study are as follows. Although the lateral ventricle segmentation scheme proposed in this study achieves relatively high segmentation accuracy compared with other algorithms, the morphological operation used in the segmentation scheme to improve the accuracy will inevitably lead to the omission of segmentation of some regions in the posterior or lower corner of the lateral ventricle in individual images. Therefore, statistical analysis can be used to further correct the segmentation results. For certain brain diseases, the corresponding tissue will change accordingly, so the automatic segmentation of different tissue has great significance for the diagnosis of brain diseases. However, the existing segmentation schemes are all aimed at the segmentation of a certain region, which is not suitable for the segmentation of other brain regions. Therefore, in the future, adaptive segmentation of different regions can be carried out according to the required regions in the process of lateral ventricle segmentation combined with the overall information of gray image.

\section{Conclusion}

The study focused on the relationship between changes in the flow of cerebrospinal fluid and communicating hydrocephalus after decompressive craniectomy for craniocerebral injury, and the dynamics of cerebrospinal fluid flow in the cerebral aqueducts was analyzed. The results showed that the third ventricle of the study group was significantly reduced, and the flow of cerebrospinal fluid was similar to that of normal people. It suggested that decompressive craniectomy can relieve communicating hydrocephalus, and the flow rate of cerebrospinal fluid is associated with the occurrence of hydrocephalus.

\section{Data Availability}

The data used to support the findings of this study are available from the corresponding author upon request.

\section{Conflicts of Interest}

The authors declare that there are no conflicts of interest.

\section{References}

[1] E. Meng, Y. Duan, and X. Wang, "Therapeutic mechanism of intracranial infection in patients with hydrocephalus after craniocerebral injury based on decompressive craniectomy," Saudi Journal of Biological Sciences, vol. 27, no. 3, pp. 873-880, 2020.

[2] K. H. Chen, C. P. Lee, Y. H. Yang et al., "Incidence of hydrocephalus in traumatic brain injury: a nationwide population-based cohort study," Medicine, vol. 98, no. 42, Article ID e17568, 2019.

[3] A. Harbert, K. Bradford, V. Ritter, W. T. Northam, and C. Quinsey, "National imaging trends in pediatric traumatic brain injury and hydrocephalus," World Neurosurgery, vol. 139, pp. e399-e405, 2020.

[4] A. Harbert, W. Northam, S. Elton, and C. Quinsey, "Targeted head CT reduction for pediatric patients with hydrocephalus and traumatic brain injury: academic center institutional experience as an example of opportunities for further improvement," Child's Nervous System, vol. 36, no. 3, pp. 583-589, 2020.

[5] P. De Bonis and C. Anile, "Post-traumatic hydrocephalus: the cinderella of neurotrauma," Expert Review of Neurotherapeutics, vol. 20, no. 7, pp. 643-646, 2020.

[6] M. N. Cizmeci, N. Khalili, N. H. P. Claessens et al., "Assessment of brain injury and brain volumes after posthemorrhagic ventricular dilatation: a nested substudy of the randomized controlled elvis trial," The Journal of Pediatrics, vol. 208, pp. 191-197.e2, 2019.

[7] G. W. J. Hawryluk, A. M. Rubiano, A. M. Totten et al., "Guidelines for the management of severe traumatic," Brain Injury, vol. 87, no. 3, pp. 427-434, 2020.

[8] D. A. Brown and E. F. Wijdicks, "Decompressive craniectomy in acute brain injury," Handbook of Clinical Neurology, vol. 140, pp. 299-318, 2017.

[9] C. Lambride, N. Christodoulou, A. Michail, V. Vavourakis, and T. Stylianopoulos, "Decompressive craniectomy of posttraumatic brain injury: an in silico modelling approach for intracranial hypertension management," Scientific Reports, vol. 10, no. 1, p. 18673, 2020.

[10] S. W. Bothwell, D. Janigro, and A. Patabendige, "Cerebrospinal fluid dynamics and intracranial pressure elevation in neurological diseases," Fluids and Barriers of the CNS, vol. 16, no. 1, p. 9, 2019.

[11] K. B. Mahaney, C. Buddhala, M. Paturu, D. Morales, D. D. Limbrick, and J. M. Strahle, "Intraventricular hemorrhage clearance in human neonatal cerebrospinal fluid: associations with hydrocephalus," Stroke, vol. 51, no. 6, pp. 1712-1719, 2020.

[12] W. C. Shen and J. T. Ho, "Technology-enhanced learning in higher education: a bibliometric analysis with latent semantic 
approach," Computers in Human Behavior, vol. 104, Article ID 106177, 2020.

[13] H. C. Bock, S. F. Dreha-Kulaczewski, A. Alaid, J. Gärtner, and H. C. Ludwig, "Upward movement of cerebrospinal fluid in obstructive hydrocephalus-revision of an old concept," Childs Nerv Syst, vol. 35, no. 5, pp. 833-841, 2019.

[14] P. Krishnan, C. Raybaud, S. Palasamudram, and M. Shroff, "Neuroimaging in pediatric hydrocephalus," Indian J Pediatr, vol. 86, no. 10, pp. 952-960, 2019.

[15] P. T. Akins and K. H. Guppy, "Are hygromas and hydrocephalus after decompressive craniectomy caused by impaired brain pulsatility, cerebrospinal fluid hydrodynamics, and glymphatic drainage? Literature overview and illustrative cases," World Neurosurg, vol. 130, pp. e941-e952, 2019.

[16] R. Spaull, B. McPherson, A. Gialeli et al., "Exosomes populate the cerebrospinal fluid of preterm infants with post-haemorrhagic hydrocephalus," International Journal of Developmental Neuroscience, vol. 73, pp. 59-65, 2019.

[17] B. Atasoy, A. Aralasmak, E. Cetinkaya et al., "Normal pressure hydrocephalus: clinical symptoms, cerebrospinal fluid flow metrics and white matter changes," Journal of Computer Assisted Tomography, vol. 44, no. 1, pp. 59-64, 2020.

[18] Q. Zeng, G. Samei, D. Karimi et al., "Prostate segmentation in transrectal ultrasound using magnetic resonance imaging priors," International Journal of Computer Assisted Radiology and Surgery, vol. 13, no. 6, pp. 749-757, 2018.

[19] A. Danelakis, T. Theoharis, and D. A. Verganelakis, "Survey of automated multiple sclerosis lesion segmentation techniques on magnetic resonance imaging," Computerized Medical Imaging and Graphics, vol. 70, pp. 83-100, 2018.

[20] Y. Chang, "Improving the Otsu method for MRA image vessel extraction via resampling and ensemble learning," Healthc Technol Lett, vol. 6, no. 4, pp. 115-120, 2019.

[21] D. R. Roberts and L. G. Petersen, "Studies of hydrocephalus associated with long-term spaceflight may provide new insights into cerebrospinal fluid flow dynamics here on earth," JAMA Neurology, vol. 76, no. 4, pp. 391-392, 2019.

[22] A. Agarwal, G. Bathla, and S. Kanekar, "Imaging of communicating hydrocephalus," Seminars In Ultrasound CT and $M R$, vol. 37, no. 2, pp. 100-108, 2016.

[23] K. Zhao, H. Sun, Y. Shan, B. Y. Mao, and H. Zhang, "Cerebrospinal fluid absorption disorder of arachnoid villi in a canine model of hydrocephalus," Neurology India, vol. 58, no. 3, pp. 371-376, 2010.

[24] F. Godenschweger, U. Kägebein, D. Stucht et al., "Motion correction in MRI of the brain," Physics in Medicine and Biology, vol. 61, no. 5, pp. R32-R56, 2016. 